\title{
Effects of gravity level on bubble formation and rise in low-viscosity liquids
}

\author{
Francesc Suñol and Ricard González-Cinca \\ Universitat Politècnica de Catalunya-BarcelonaTech, Department of Applied Physics, \\ c/ E. Terradas, 5, 08860 Castelldefels (Barcelona), Spain
}

(Dated: June 30, 2015)

\begin{abstract}
We present an experimental analysis of the effects of gravity level on the formation and rise dynamics of bubbles. Experiments were carried out with millimetre-diameter bubbles in the hypergravity environment provided by the Large Diameter Centrifuge of the European Space Agency. Bubble detachment from a nozzle is determined by buoyancy and surface tension forces regardless of the gravity level. Immediately after detachment, bubble trajectory is deviated by the Coriolis force. Subsequent bubble rise is dominated by inertial forces and follows a zig-zag trajectory with amplitude and frequency dependent of the gravity level. Vorticity production is enhanced as gravity increases, which destabilizes the flow and therefore the bubble path.

PACS numbers: 47.55.dd, 47.55.P-, 47.20.Ky
\end{abstract}

The control of bubble dynamics is an important issue for the improvement of the performance of many industrial applications containing bubbly flows. The formation and rise of bubbles are fundamental phenomena in two-phase flows management that attract continuous attention from researchers $[1,2]$.

The bubble formation process determines the bubble size depending on the physicochemical properties of the two-phase system and the nozzle configuration [1]. After detachment from the nozzle, bubbles accelerate upwards following an initial vertical path. Depending on the bubble and liquid properties, the path can become unstable in a very short time. The physical causes of path instability can be divided into two classes [2]. On the one hand, they can be related to the way the hydrodynamical forces and torques evolve when a disturbance is applied to the bubble degrees of freedom. On the other hand, the origin of the instability is associated to the wake instability that occurs in some parameter regimes. The path instability can result in different types of trajectories, like zig-zag or helical paths. The zig-zag trajectory is confined in a two-dimensional vertical plane, whereas the helical one combines a constant vertical rise and a circular, elliptical or spiral horizontal motion. For air bubbles rising in water, spiral or zig-zag paths were found only for bubbles with equivalent diameter $d_{e} \geq 1.5 \mathrm{~mm}$, while smaller bubbles rose rectilinearly $[3,4]$. Bubble shape along these trajectories remains close to an oblate spheroid [3-5].

Experiments performed by Wu and Gharib [4] showed that a bubble formed by inflating it at a nozzle will usually show an ellipsoidal shape due to the perturbation in the interface generated by the pinch-off event, and rise in a helical or spiral path. If the bubble is gently released from the nozzle can maintain a spherical shape with a lower rise velocity $U$ with respect to the ellipsoidalshaped bubble. These spherical bubbles rise rectilinearly or in a zig-zag motion.

Zenit and Magnaudet [6] found that the dominant parameter to trigger the instability is the bubble shape and not the Reynolds number, due to the fact that vorticity generated at the bubble surface is almost independent of the Reynolds number and mostly depends on the bubble aspect ratio.

In the wake-induced zig-zag path of rising bubbles, the flow bifurcates from an axisymmetric shape to a pair of long thin vortices located at the trailing edge of the bubble. These vortices rotate in opposite directions and their rotation axis is aligned parallel to the bubble path $[3,7-$ 10]. The flow becomes unstable once the amount of vorticity produced at the bubble surface exceeds a critical value [10-12]. Zig-zag has been associated to the periodical switch of sign of the axial vorticity components of the double-threaded wake $[3,13]$. It has been suggested that wake vortices induce a lift force perpendicular to the bubble motion, which produces an oscillating trajectory $[5,10,14]$. The crucial role played by the lift force in the oscillatory path instabilities has been confirmed in numerous experiments [5, 9, 15-17].

Experimental studies on bubble formation and rise were carried out so far in normal gravity or, in much lesser occasions, in microgravity conditions. In spite of the main role played by buoyancy force in these phenomena, no experimental work exploring higher gravity levels were performed. In this Letter we present an experimental study on the bubble formation and inertial rise in a hypergravity environment. The role played by the gravity level in the path instability and in the oscillatory trajectory characteristics is analyzed.

An experimental setup has been designed for its use at the ESA Large Diameter Centrifuge (LDC) in Noordwijk (The Netherlands). This platform can provide hypergravity levels from $1 g_{0}$ up to $20 g_{0}$, where $g_{0}=9.81$ $\mathrm{m} / \mathrm{s}^{2}$ is the acceleration due to gravity at sea level. The LDC uses the centrifugal force as a source of artificial gravity to provide a hypergravity environment. To this end, the experiment is placed in a gondola that can spin at an angular velocity $\omega_{L D C}$ at a distance $R_{L D C}=4$ $\mathrm{m}$ from the rotation axis of the LDC. The gondola can 
also rotate with respect to the horizontal arm of the LDC from which is suspended. Therefore, the gondola is inclined an angle $\alpha$ with respect to the real gravity vector, remaining the gondola's floor always perpendicular to the artificial gravity. In the experimental setup, air is injected into a distilled water (density $\rho=998 \mathrm{~kg} / \mathrm{m}^{3}$, viscosity $\nu=10^{-6} \mathrm{~m}^{2} / \mathrm{s}$, surface tension $\sigma=7.28 \cdot 10^{-2}$ $\mathrm{N} / \mathrm{m}$ ) tank by means of a syringe pump with a stainless steel nozzle of internal diameter $d_{C}=0.15 \mathrm{~mm}$. When a steady gas flow rate is reached, the detachment and rise of bubbles is recorded by a video camera at $2000 \mathrm{fps}$.

Fig. 1a shows the trajectories of single bubbles at different gravity levels. Each image is composed of overlapped snapshots of a rising bubble. The bubble size is observed to decrease with increasing gravity level. In addition, the deviation from a rectilinear trajectory into an oscillating path is visible in most cases, being the complete wavelength observable at $g \geq 11 g_{0}$. As the gravity level is increased, the frequency of the oscillating path increases and the amplitude slightly decreases. A zigzag path lying in the $y=0$ plane is assumed throughout the paper based on three observations. First, the vertical velocity $v_{z}$ in a helical path (circular or elliptical motion in the $x-y$ plane, therefore with non-zero $v_{y}$ ) is constant. In a zig-zag path $v_{z}$ is a sinusoidal function of $z$, in which the maxima of $v_{z}$ are located in the regions where the bubble rises vertically, as observed in our measurements. Second, in case the path was helical, the projection of the bubble shape into the camera focal plane $(y=0)$ would produce a slightly variable bubble shape. In the case of a zig-zag path, the shape projection of a bubble remains constant, as observed in the analyzed videos. Third, if the bubble followed a helical path, the images obtained when the bubble was not in the $y=0$ plane would become blurred, which was not observed.

The shape of the rising bubbles can be approximated as an oblate spheroid with major axis $d_{h}$ and minor axis $d_{v}$. The equivalent diameter $d_{e}=\left(d_{h}^{2} d_{v}\right)^{\frac{1}{3}}$ corresponds to the diameter of a spherical bubble containing the same volume $V$ as the oblate spheroid. If inertial effects are neglected, the bubble size during air injection and at detachment is determined by the competition between the buoyancy force $F_{B}=\rho g V$ and the capillary force $F_{\sigma}=\pi d_{C} \sigma$. When air is injected $F_{B}$ increases linearly with time until a critical diameter is reached when $F_{B}=F_{\sigma}$ and detachment takes place. Therefore, the dependence of the equivalent diameter on the gravity level is given by:

$$
d_{e}=\left(\frac{6 \sigma d_{C}}{\rho g}\right)^{\frac{1}{3}}
$$

Fig. 2a shows the experimental values of the equivalent, horizontal, and vertical diameters as a function of the gravity level. The good agreement between the experimental data and theoretical prediction (represented (a)

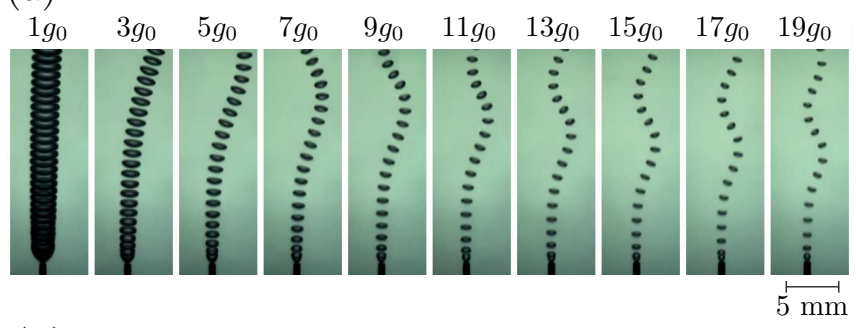

(b)
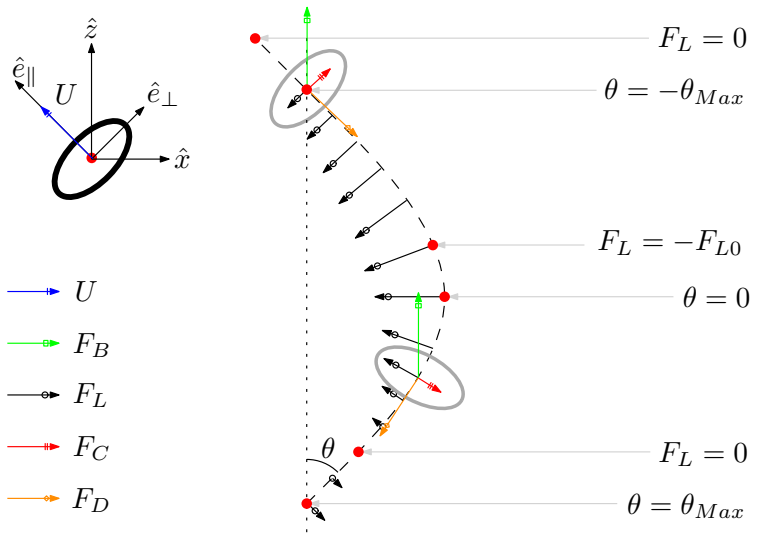

FIG. 1. a) (Color online) Series of overlapped snapshots of an air bubble rising at different gravity levels. Time interval between snapshots is $5 \mathrm{~ms}$. b) Sketch of half wavelength of the zig-zag path and acting forces. $U$ : rise velocity; $F_{B}$ : buoyancy force; $F_{L}$ : lift force; $F_{C}$ : Coriolis force; $F_{D}$ : drag force.

by a solid line in the figure) of $d_{e}$ confirms that inertial effects can be neglected during the detachment process in the present experiments. The capillary length, $C_{\ell}=\sqrt{\sigma /(\rho g)}$ (dashed line in the figure), is closer to $d_{e}$ at larger gravity levels.

Once bubbles detach from the nozzle, the initial vertical trajectory of all of them is deviated towards the same direction (to the right in Fig. 1a). The trigger of this path instability can be attributed to the Coriolis force $\left(F_{C}=2 \rho V U \omega_{L D C} \sin \alpha\right.$, where $U$ is the rise velocity, $\omega_{L D C}=\left(\frac{\sqrt{g^{2}-g_{0}^{2}}}{R_{L D C}}\right)^{\frac{1}{2}}$, and $\alpha=\arctan \left(\frac{\sqrt{g^{2}-g_{0}^{2}}}{g_{0}}\right)$ is the angle between $U$ and $\omega_{L D C}$ ), which is perpendicular to the bubble velocity. However, $F_{C}$ can not be the responsible for the whole zig-zag path. This oscillatory trajectory is observed at $g>g_{0}$ in bubbles with $d_{e}<1.5 \mathrm{~mm}$ (Fig 2a), in opposition to the rectilinear rise of bubbles of this size observed in experiments at $g_{0}[3,4]$.

In order to understand the trajectory shown by the bubbles, the forces acting on them must be considered. Fig. 1b shows these forces in a section of the trajectory: $F_{B}, F_{C}$, lift force $F_{L}$, and drag force $F_{D}$. The generalized Kirchhoff equations can be used to describe the motion of a non-deformable body moving in an ideal fluid $[5,14]$. In the present experiments, bubble dynamics is 
mainly controlled by inertial forces. The Reynolds number $\left(\operatorname{Re}=U d_{e} / \nu\right)$ ranges from 323 to 617 .

Considering a frame of reference moving with the bubble, where $\hat{e}_{\|}$and $\hat{e}_{\perp}$ are the unit vectors in the axis parallel and perpendicular to the bubble motion, respectively (Fig. 1b), the equations of motion can be written as:

$$
\begin{gathered}
\mathcal{A}_{11} \frac{d U}{d t}=F_{B_{\|}}-F_{D} \\
\mathcal{A}_{11} U \frac{d \theta}{d t}=F_{L}+F_{C}-F_{B_{\perp}},
\end{gathered}
$$

where $\theta$ is the angle between $\hat{e}_{\|}$and $\hat{z}, F_{B_{\|}}=$ $\rho g V \cos \theta, F_{B_{\perp}}=\rho g V \sin \theta, F_{D}=\frac{1}{2} \rho C_{D} S U^{2}$, where $C_{D}$ is the drag coefficient and $S=\pi d_{h}^{2} / 4$ is the projected area, $\mathcal{A}_{11}=C_{M} \rho V$, and $C_{M}=\frac{\gamma}{2-\gamma}$ with $\gamma=$ $\frac{2}{e^{2}}\left[1-\frac{\sqrt{1-e^{2}}}{e} \arcsin (e)\right]$ is the added mass coefficient of an oblate spheroid, with $e=\sqrt{1-\epsilon^{-2}}, \epsilon$ being the aspect ratio.

Eqs. 2 and 3 can be rewritten in the following form:

$$
\begin{aligned}
& a_{\|}=\frac{d U}{d t}=\frac{g \cos \theta}{C_{M}}-\frac{3 C_{D} U^{2}}{4 C_{M} d_{e} \epsilon^{-2 / 3}} \\
& a_{\perp}=U \frac{d \theta}{d t}=-\frac{g \sin \theta}{C_{M}}+\frac{F_{L}+F_{C}}{\rho V C_{M}} .
\end{aligned}
$$

Theoretical or quantitative models are available for the forces playing a role in the bubble dynamics. $F_{L}$ is usually associated to the wake vortices around the bubble. Shew and Pinton [14] modelled $F_{L}$ and showed that it plays a crucial role in the oscillatory path instabilities. Assuming $F_{C}$ negligible along the zig-zag trajectory, the lift force can be expressed from Eq. 5 as:

$$
F_{L}=\rho V\left(g \sin \theta+C_{M} U \frac{d \theta}{d t}\right)
$$

which corresponds to a periodical force with amplitude $F_{L 0}$ and the same frequency $\omega$ as in a sinusoidal bubble trajectory given by $x(z)=A \sin (k z)$, but shifted with respect to it. Fig. 1b illustrates the shift between the maxima and minima of $x$ and $F_{L}$. Assuming the sinusoidal trajectory, the dependence of the lift force on gravity can be obtained from $U, \omega$ and $A$ at different gravity levels.

Experimental measurements show that the rise velocity is constant along the zig-zag trajectory and increases with the gravity level (Fig. 2b). Taking into account that the vertical velocity $v_{z}(z)$ (do not confuse it with the rise velocity $U$ ) is a sinusoidal function with maximum values at $\theta=0$ (vertical rise) and the sinusoidal dependence of $x$ on $z, U$ should be constant. Considering Eq. 4 and the variation of $\theta$ along the trajectory, the aspect ratio $\epsilon$ is expected to change, in agreement with previous works $[13,18]$. However, a fixed bubble shape can be assumed when Kirchhoff equations are applied [14], and, in particular, when Eq. 6 is used to determine $F_{L}$.

An expression for the rise velocity of a non-spherical bubble can be determined by considering it as a disturbance on the gas-liquid interface propagating through the liquid in the form of a gravity and surface tension wave $[19,20]$. From this model, the bubble rise velocity is directly related to gravity and the bubble equivalent diameter, $U=\sqrt{2 \sigma /\left(\rho d_{e}\right)+g d_{e} / 2}$, which, substituting Eq. 1 in it, leads to:

$$
U=\left[\left(\frac{4 \sigma^{2} g}{3 \rho^{2} d_{C}}\right)^{\frac{1}{3}}+\left(\frac{3 \sigma d_{C} g^{2}}{4 \rho}\right)^{\frac{1}{3}}\right]^{\frac{1}{2}} .
$$

The dependence of the rise velocity with gravity expressed in Eq. 7 is represented in Fig. 2b by a solid line. Good agreement between the experimental results and the model is specially found at the lowest gravity levels. The slight discrepancy observed at large gravity could be explained by a limitation in the validity of the model when the bubble trajectory diverges from a vertical path.

Fig. 2c shows the frequency of the zig-zag trajectory as a function of the gravity level. The frequency of the vortex shedding from the rear part of a spheroidal body is $f_{s}=p \nu \operatorname{Re} /\left(\pi d_{e}^{2}\right)$, where Re is the Reynolds number and $p$ is a parameter that depends on the shape of the body ( $p=0.3$ for a rigid sphere) [21,22]. Combining the latter expression with Eqs. 1 and 7, we obtain:

$$
\omega=2 \pi f_{s}=2 p \sqrt{\frac{g}{3 d_{C}}+\left(\frac{\rho g^{4}}{48 d_{C} \sigma}\right)^{\frac{1}{3}}} .
$$

The solid line in Fig. 2c shows the fit of Eq. 8 to experimental data with $p=0.265 \pm 0.003$, which indicates a bubble shape slightly deviated from a sphere.

Fig. 2d shows the amplitude $A$ of the zig-zag trajectory as a function of the gravity level. Solid line corresponds to the fit of $A=C\left(g / g_{0}\right)^{n}$ to experimental data with $C=$ $2.94 \pm 0.23$ and $n=-0.40 \pm 0.04$. The amplitude of the trajectory is related to the maximum inclination angle $\theta_{\text {Max }}$, which is found to be $\theta_{\text {Max }} \approx 30^{\circ}$ independently of the gravity level in the experiment. A prediction for the dependence of $\theta_{\text {Max }}$ with gravity can be derived from the behavior of $A, \omega$ and $U$. Since $\theta=\arctan (d x / d z)=$ $\arctan (k A \cos k z)$, and $k=2 \pi / \lambda \approx \omega / U, \theta_{\text {Max }}$ is found to lie between $23.5^{\circ}$ (at $1 g_{0}$ ) and $28.0^{\circ}$ (at $19 g_{0}$ ). The experimental uncertainty for the measurement of $\theta_{\text {Max }}$ $\left(\delta \theta_{\text {Max }}= \pm 5^{\circ}\right)$ can explain the discrepancy between the measured value and the prediction. However, both data are within the range $\theta_{\operatorname{Max}} \in\left[20^{\circ}, 30^{\circ}\right]$ reported in [10]. 


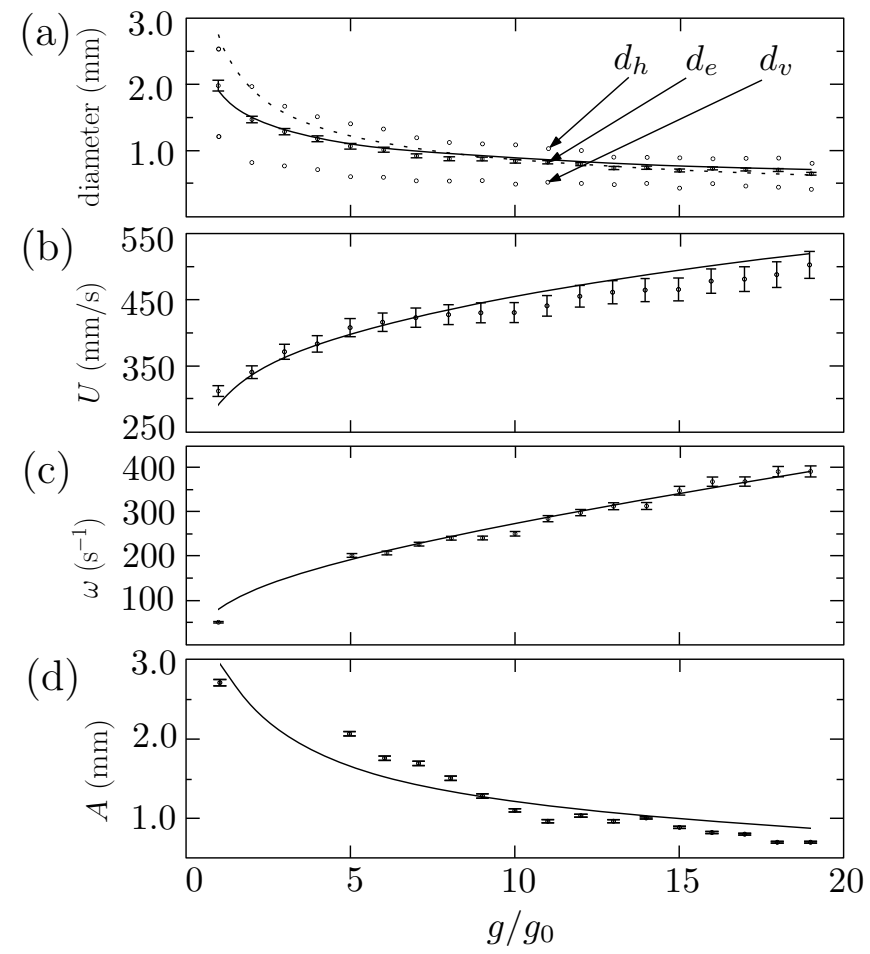

FIG. 2. a) Bubble diameters as a function of gravity level. Points: experimental data $\left(d_{h}, d_{v}\right.$, and $\left.d_{e}\right)$. Solid line: Eq. 1. Dashed line: Capillary length. b) Rise velocity as a function of the gravity level, solid line corresponds to Eq. 7; c) Frequency of the zig-zag trajectory, solid line corresponds to Eq. 8; d) Amplitude of the zig-zag trajectory, solid line corresponds to a power fit. In c) and d), measurements at $1<g / g_{0}<5$ are omitted since less than a half-wavelength of oscillation occurred within the field of view.

The fit of the experimental data of $U, \omega$ and $A$ have been used to numerically determine the lift force by means of Eq. 6. The amplitude of $F_{L}$ obtained is one order of magnitude larger than $F_{C}$, which confirms the negligible effects of the Coriolis force along the zig-zag path. Fig. 3a shows the amplitude of the lift acceleration $a_{L 0}$ as a function of the gravity level. The acceleration increases with gravity despite $F_{L}$ decreases due to the decrease in the bubble size. Lift force arises from wake vortices that develop when the vorticity generated on the bubble surface is sufficiently large $[3,5,7,10]$. The maximum vorticity $\tilde{\omega}_{\max }$ produced can be obtained from [11]:

$$
\tilde{\omega}_{\max }=\frac{4 U}{d_{e}} \frac{\epsilon^{5 / 3}\left(\epsilon^{2}-1\right)^{3 / 2}}{\epsilon^{2} \sec ^{-1} \epsilon-\left(\epsilon^{2}-1\right)^{1 / 2}} .
$$

When $\tilde{\omega}_{\text {max }}$ is larger that a critical vorticity given by $\tilde{\omega}_{c}(\operatorname{Re}) d_{e} /(2 U) \approx 12.5+4.3 \cdot 10^{-3} \operatorname{Re}$, the flow is unstable and a path instability takes place [11].

Fig. 3b shows the maximum and critical vorticity obtained from the experimental data as a function of the gravity level. In all cases the vorticity generated is so
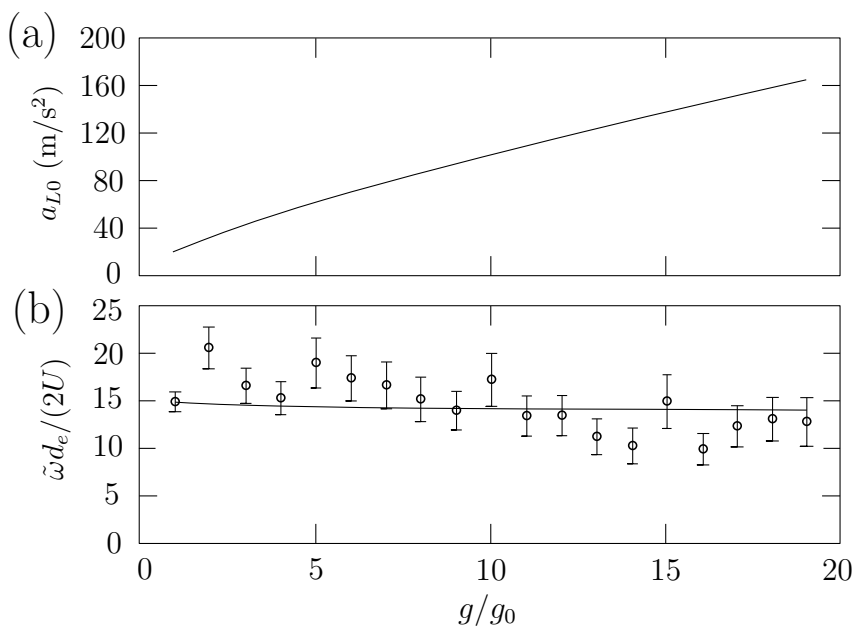

FIG. 3. a) Amplitude of the lift acceleration as a function of the gravity level, b) Maximum (dots, Eq. 9) and critical (line) vorticity as a function of the gravity level.

high that it can not be evacuated downstream. Hence, the axisymmetric wake becomes unstable and breaks into a double-threaded open wake, which explains the zig-zag trajectory observed at $g>g_{0}$ for sizes of bubbles that would rise rectilinearly at $g_{0}$.

Fig. 3c shows the maximum vorticity as a function of the equivalent diameter for rise at $g_{0}$ and at $g>g_{0}$. Bubbles generate more vorticity at larger gravity levels, therefore they can rise rectilinearly at $g_{0}$ and in zig-zag at $g>g_{0}$.

In conclusion, the formation and rise of a bubble in the hypergravity environment provided by the LDC presents some unique features. For a fixed nozzle diameter, the bubble size is determined by the balance between gravity and surface tension forces. The bubble trajectory after detachment is deviated by the Coriolis force. Subsequent bubble rise takes place in an oscillatory trajectory at all gravity levels considered. As gravity is increased, trajectories with larger frequency and smaller amplitude are observed. The vorticity produced at the bubble surface grows with gravity and is sufficiently large to generate a wake instability that destabilizes the flow and induces the zig-zag trajectory. Coriolis force does not play any significant role in the zig-zag. Although bubbles of the considered size in $g>g_{0}$ rise rectilinearly in normal gravity, at larger gravity levels more vorticity is produced along the path, which generates the observed instability.

This research was supported by the Dirección General de Investigación Científica y Técnica of the Spanish Ministerio de Economía y Competitividad (Project AYA2012-34131), and was carried out in the framework of the EU COST Action MP1106. We acknowledge the ESA Education Office for the Spin Your Thesis 2011! Campaign. 
[1] A. A. Kulkarni and J. B. Joshi, Ind. Eng. Chem. Res. 44, 5873 (2005).

[2] P. Ern, F. Risso, D. Fabre, and J. Magnaudet, Annu. Rev. Fluid Mech. 44, 97 (2012).

[3] A. W. G. de Vries, A. Biesheuvel, and L. van Wijngaarden, Int. J. Multiph. Flow 28, 1823 (2002).

[4] M. Wu and M. Gharib, Phys. Fluids 14, L49 (2002).

[5] W. L. Shew, S. Poncet, and J.-F. Pinton, J. Fluid Mech. 569, 51 (2006).

[6] R. Zenit and J. Magnaudet, Phys. Fluids 20 (2008).

[7] K. Lunde and R. J. Perkins, Proceedings of the ASME FED Summer Meeting. Vancouver, Canada, Paper FEDSM'97-3530 (1997).

[8] C. Brucker, Phys. Fluids 11, 1781 (1999).

[9] K. Ellingsen and F. Risso, J. Fluid Mech. 440, 235 (2001).

[10] G. Mougin and J. Magnaudet, Phys. Rev. Lett. 88, 014502 (2002).

[11] J. Magnaudet and G. Mougin, J. Fluid Mech. 572, 311
(2007).

[12] B. Yang and A. Prosperetti, J. Fluid Mech. 582, 53 (2007).

[13] A. W. G. de Vries, PhD dissertation, University of Twente, The Netherlands (2001).

[14] W. L. Shew and J.-F. Pinton, Phys. Rev. Lett. 97, 144508 (2006).

[15] M. Rastello, J.-l. Mari, N. Grosjean, and M. Lance, J. Fluid Mech. 624, 159 (2009).

[16] M. Rastello, J.-l. Mari, and M. Lance, J. Fluid Mech. 682, 434 (2011).

[17] J. Tchoufag, J. Magnaudet, and D. Fabre, J. Fluid Mech. 751 (2014), 10.1017/jfm.2014.340.

[18] D. Legendre, R. Zenit, and J. R. Velez-Cordero, Phys. Fluids 24, 043303 (2012).

[19] H. D. Mendelson, A. I. Ch. E. J. 13, 250 (1967).

[20] A. Tomiyama, I. Kataoka, I. Zun, and T. Sakaguchi, JSME Int. J. Series B 41, 472 (1998).

[21] I. Kim and A. J. Pearlstein, J. Fluid Mech. 211, 73 (1990).

[22] Y. Tagawa, S. Takagi, and Y. Matsumoto, J. Fluid Mech. 738, 124 (2014). 